\title{
AVALIAÇÃO DA SATISFAÇÃO DOS USUÁRIOS DE UM SERVIÇO DE SAÚDE PÚBLICO-PRIVADO NO NORDESTE DO BRASIL E A JUDICIALIZAÇÃO DA SAÚDE
}

Evaluation of patient's satisfaction in a public-private health facility in Northeastern Brazil and the judicialization of healthcare

${ }^{1}$ Universidade Federal da Bahia. Salvador/BA, Brasil.

${ }^{2}$ Universidade de Fortaleza. Fortaleza/CE, Brasil.

Correspondência: Geraldo Bezerra da Silva Junior. E-mail: geraldobezerrajr@yahoo.com.br.

Recebido em: 18/05/2015. Revisado em: 17/08/2015. Aprovado em: 19/08/2015. 


\section{RESUMO}

Este artigo tem por escopo analisar o grau de satisfação dos usuários de um serviço público-privado de saúde do Nordeste do Brasil, bem como o nível de conhecimento sobre seus direitos e a procura pelo Judiciário para garanti-los. Foram avaliadas as respostas dadas a um questionário aplicado junto a 67 pacientes atendidos pelo Núcleo de Assistência Médica Integrada, da Universidade de Fortaleza, conveniado ao Sistema Único de Saúde (SUS). Os pacientes entrevistados mostraram satisfação com os serviços oferecidos, considerando-os como bons ou excelentes. O principal problema evidenciado foi a dificuldade de acesso a medicações fornecidas pelo SUS e a falta de conhecimento dos direitos relacionados à saúde. Observou-se uma baixa procura pela Justiça para questões ligadas à saúde, o que pode se explicar, por um lado, pelo reduzido grau de conhecimento dos direitos relacionados a tal campo e pela baixa escolaridade da maior parte dos entrevistados. A melhora do grau de satisfação pode contribuir para reduzir a judicialização, muito embora usuários mais bem informados possam se mostrar mais exigentes e recorrer mais à Justiça. Soluções alternativas de resolução de conflitos, como a mediação e a justiça restaurativa, podem contribuir para diminuir a busca pelo Judiciário.

\section{Palavras-Chave}

Direito à Saúde; Judicialização da Saúde; Satisfação dos Usuários de Saúde.

\section{ABSTRACT}

This article aims to analyze the degree of satisfaction of users of a public-private healthcare service in the Northeast of Brazil, as well as their level of knowledge about their rights and about the judicial procedure used to guarantee these rights. The analysis was based on the responses to a questionnaire applied to 67 patients who are users of a healthcare assistance center that is part of the Brazilian National Public Health System, at Universidade de Fortaleza. The users showed satisfaction with the services offered, seeing them as good or excellent. The main problem highlighted was the difficulty of access to medications provided by the public health system and the lack of knowledge on health-related rights. There was a low demand for justice related to health issues; this can be explained by both the low level of knowledge on the rights related to this field and the low educational level of most respondents. The improvement of the degree of satisfaction can help reduce the judicialization, even though more knowledgeable users might become more demanding and appeal more to justice. Alternative solutions for resolving disputes, such as mediation and restorative justice, can help reduce the appeal to justice.

\section{Keywords}

Judicialization; Health Services Users' Satisfaction; Right to Health. 


\section{Introdução}

O Sistema Único de Saúde (SUS) tem o objetivo de prover atenção abrangente e universal, preventiva e curativa, por meio da gestão e prestação descentralizadas de serviços de saúde, promovendo a participação da comunidade em todos os níveis de governo ${ }^{1}$, sendo um dos maiores sistemas públicos de saúde do mundo e o único a garantir assistência integral e gratuita para a totalidade da população.

A prestação de serviços de saúde é também livre para a iniciativa privada, tendo, porém, relevância pública - na forma do artigo 197 da Constituição -, atuando o Estado como regulador e fiscalizador. Nesse caso, mostra-se evidente o estabelecimento de relações triangulares, em que, além do Estado e dos indivíduos que buscarão serviços e prestações na área de saúde, comparecem sujeitos privados que prestam os mencionados serviços e que também são detentores de direitos fundamentais, notadamente ligados à "manutenção da higidez econômica do mercado"

A satisfação dos usuários dos serviços de saúde, tanto públicos quanto privados, representa uma preocupação no mundo todo ${ }^{3}$. A avaliação da satisfação dos pacientes com os serviços de saúde é importante para que se conheçam melhor as falhas e os pontos positivos de cada serviço, de modo a obter subsídios para a melhoria dos serviços e para o melhor planejamento das políticas públicas voltadas ao setor da saúde. Alguns estudos têm sido realizados nesse sentido, com resultados diversos. Segundo Savassi,

A percepção do usuário interferirá na acessibilidade, na criação de vínculos e na consequente horizontalidade do cuidado, e terá reflexos na capacidade da equipe para coordenar o cuidado. [...] Os usuários geralmente demonstram maiores índices de satisfação em relação a médicos e menores em relação a exames; geralmente, a insatisfação se relaciona à medicação fornecida nas unidades ou na demora em serviços de propedêutica.

\footnotetext{
1PAIM, Jairnilson; TRAVASSOS, Claudia; ALMEIDA, Célia; BAHIA, Lígia; MACINKO, James. The Brazilian health system: history, advances, and challenges. The Lancet, London, v. 377, n. 9779, p. 1778-1797, 2011. Disponivel em: <http://www.thelancet.com/pdfs/journals/lancet/PIIS0140-6736(11)60054-8.pdf>. http://dx.doi.org/10.1016/S0140-6736(11)60054-8.

${ }^{2}$ FIGUEIREDO, Leonardo Vizeu. Curso de direito de saúde suplementar: manual jurídico de planos e seguros de saúde. 2. ed. Rio de Janeiro: Gen/Forense, 2012. p. 8.

${ }^{3}$ KRAVITZ, Richard. Patient satisfaction with health care: critical outcome or trivial pursuit? Journal of General Internal Medicine, Philadelphia, v. 13, n. 4, p. 280-282, Apr. 1998; ERVIN, Naomi. Does patient satisfaction contribute to nursing care quality? Journal of Nursing Administration, Philadelphia, v. 36, n. 3, p. 126-130, 2006; HAWTHORNE, Graeme; SANSONI, Jan; HAYES, Laura; MAROSSZEKY, Nick; SANSONI, Emily. Measuring patient satisfaction with health care treatment using the Short Assessment of Patient Satisfaction measure delivered superior and robust satisfaction estimates. Journal of Clinical Epidemiology, New York, v. 67, n. 5, p. 527-537, 2014. 10.1016/j.jclinepi.2013.12.010; MERCER, Mary; HERNANDEZ-BOUSSARD, Tina; MAHADEVAN, Swaminatha; STREHLOW, Matthew. Physician identification and patient satisfaction in the emergency deparment: are they related? Journal of Emergency Medicine, New York, v. 46, n. 5, p. 711-718, May 2014. doi: 10.1016/j.jemermed.2013.08.036; SAVASSI, Leonardo Cançado Monteiro. A satisfação do usuário e a autopercepção da saúde em atenção primária. Revista Brasileira de Medicina de Família e Comunidade, Rio de Janeiro, v. 5, n. 17, p. 3-5, 2010. Disponível em: <https://www.rbmfc.org.br/rbmfc/ article/view/135/127>. http://dx.doi.org/10.5712/rbmfc5(17)135.
} 
Também se pode estabelecer uma relação entre o grau de satisfação e o eventual recurso ao Poder Judiciário para obtenção de tratamentos e medicamentos. Não se pode afastar a existência de uma maior propensão à busca de medidas judiciais por quem não se está satisfeito com os serviços oferecidos. E, quanto maior o grau de conhecimento de seus direitos, maior tende a ser o nível de exigência e, em consequência, a busca do Judiciário para suprir as falhas dos serviços de saúde.

O objetivo deste estudo é avaliar o grau de satisfação dos usuários de um serviço de saúde público-privado no Nordeste do Brasil e em que medida eles procuram o Judiciário para garantir os direitos relacionados à saúde.

\section{Metodologia}

Foi realizado estudo transversal, por meio de entrevista, com usuários do Núcleo de Assistência Médica Integrada (NAMI) da Universidade de Fortaleza (Unifor), conveniado ao Sistema Único de Saúde (SUS), localizado na cidade de Fortaleza, Ceará, Brasil, no período de julho a novembro de 2014. O protocolo do estudo foi revisado e aprovado pelo comitê de ética em pesquisa envolvendo seres humanos da Unifor (Parecer n. 545.100).

O NAMI, fundado em 1978 e ampliado em 2004, é um serviço de atenção à saúde de nível secundário, mantido pela Unifor e conveniado ao SUS. Anualmente o serviço realiza mais de 300 mil procedimentos, entre consultas médicas, análises laboratoriais, vacinas e serviços especializados em diagnóstico por imagem, enfermagem, nutrição, psicologia, fisioterapia, fonoaudiologia, serviço social e terapia ocupacional. Os pacientes são encaminhados por Unidades Básicas de Saúde da região metropolitana de Fortaleza para consulta especializada nas diversas áreas da medicina, seguindo um sistema de referência e contrarreferência da Prefeitura Municipal de Fortaleza.

Os pacientes foram entrevistados no momento em que aguardavam consulta com o médico no NAMI-Unifor. Após serem esclarecidos sobre os objetivos do estudo, os pacientes que aceitassem participar assinavam o termo de consentimento livre e esclarecido e seguiam para a entrevista. Foi destacado que o sigilo sobre suas identidades seria preservado, de forma a não expor os respondentes. Foi aplicado um questionário compreendendo perguntas sobre dados sociodemográficos e sobre o serviço de saúde em questão (NAMI), bem como sobre os conhecimentos dos pacientes acerca dos direitos relacionados à saúde e sobre a busca de sua efetivação por meios judiciais.

\section{Resultados}

Foram entrevistados 67 pacientes, com idade variando entre 21 a 89 anos (média de $47 \pm 13$ anos), sendo 15 (22,3\%) do sexo masculino e 52 (77,7\%) do sexo 
feminino. A maioria dos pacientes era procedente de Fortaleza ( $89,5 \%)$, sendo o restante (10,5\%) procedente do interior do Estado do Ceará. Com relação à escolaridade, 54 pacientes $(80,5 \%)$ tinham ensino fundamental; oito (12\%), ensino médio; e cinco (7,5\%), ensino superior. A renda média era de até quatro salários mínimos (em torno de $\mathrm{R} \$ 2.800,00$ ). Apenas dois pacientes relataram ter planos de saúde privados e um, ser assistido por plano oferecido a servidores públicos.

Os principais achados do questionário aplicado estão resumidos na Tabela 1. A primeira pergunta do questionário relacionada à saúde era sobre a satisfação do paciente quanto ao serviço de saúde oferecido pelo NAMI. Todos os entrevistados referiram estar satisfeitos com o serviço. Todos os pacientes qualificaram o serviço como bom ou excelente. Ao serem solicitados a qualificar o atendimento médico recebido, 44 (66\%) qualificaram como excelente; 21 (31\%), como bom; e dois (3\%), como regular. Foi investigado o fato de o paciente já ter recebido, em alguma ocasião, prescrição de medicação a qual não teve acesso. A maioria (61\%) referiu já ter vivenciado a experiência de não ter recebido a medicação prescrita pelo SUS; oito (11\%) responderam já ter pagado para ter acesso a procedimento ou consulta ou exame em unidade pública de saúde. Nos

Tabela 1. Avaliação da satisfação dos usuários do Núcleo de Assistência Médica Integrada da Universidade de Fortaleza no período de julho a novembro de 2014.

\begin{tabular}{lcc}
\hline & $\mathrm{n}$ & $\%$ \\
\hline Satisfação com o serviço oferecido & 67 & 100 \\
Avaliação do serviço em geral & & \\
$\quad$ Excelente & 33 & 49,3 \\
Bom & 34 & 50,1 \\
$\quad$ Regular & 0 & 0 \\
Avaliação do atendimento médico & & \\
$\quad$ Excelente & 44 & 66 \\
$\quad$ Bom & 21 & 31 \\
$\quad$ Regular & 2 & 3 \\
Falta de acesso à medicação prescrita & 41 & 61 \\
Pagamento para acesso a consulta, exame complementar ou medicamentos & 8 & 11 \\
Conhecimento dos direitos relacionados à saúde & 22 & 33 \\
Conhecimento das leis relacionadas ao direito à saúde & 8 & 11 \\
Procura do Judiciário para resolver questões ligadas à saúde & 4 & 5,9 \\
\hline
\end{tabular}


casos de pacientes com convênio (plano de saúde), nenhum afirmou já ter pagado para obter atendimento em algum serviço particular.

Com relação ao conhecimento dos direitos relacionados à saúde e assegurados pela Constituição brasileira, 45 (67\%) afirmaram não conhecê-los. Apenas oito (11\%) afirmaram conhecer as leis que regem o serviço público de saúde. Somente quatro $(5,9 \%)$ afirmaram já ter recorrido ao Judiciário para obter algum procedimento, exame ou consulta, tendo feito por meio de advogado particular (50\%) ou defensoria pública (50\%). Ao serem questionados sobre o atendimento em outros serviços, a maioria $(58,2 \%)$ não acredita que seria mais bem atendida caso estivesse em serviço privado.

Aparentemente, a baixa procura pelo Poder Judiciário se relaciona com a baixa escolaridade da maioria dos entrevistados e com o reduzido conhecimento acerca dos direitos relativos à saúde. Apesar de satisfeitos com o serviço ofertado pelo NAMI, os pacientes que responderam à pesquisa relataram ter vivenciado em outras unidades situações que ensejam normalmente o recurso ao Poder Judiciário, como a falta de acesso à medicação.

\section{Discussão}

O presente estudo investigou a opinião dos usuários de um serviço de saúde vinculado ao SUS em uma região metropolitana do Nordeste do Brasil. Observou-se que todos os pacientes estavam satisfeitos com o serviço de saúde questionado. Entretanto, alguns problemas foram apontados, como o não fornecimento de algumas medicações pelo SUS e a falta de conhecimento por parte dos pacientes dos direitos relacionados à saúde. Esse desconhecimento parece decorrer da baixa escolaridade da maioria dos respondentes. E pode explicar também por que poucos deles já recorreram ao Judiciário. Estudos recentes do Instituto de Pesquisa Econômica Aplicada (Ipea) evidenciam que a saúde (22,3\%) é um dos principais problemas enfrentados na atualidade no Brasil, de acordo com a percepção de cidadãos entrevistados, ficando atrás apenas da violência $(23 \%)$.

A falta de conhecimento é um fator a ser considerado para a efetivação do direito à saúde no Brasil. Ainda se observa um desconhecimento acerca dos direitos à saúde por grande parte dos usuários do SUS. No presente estudo, a maioria dos pacientes entrevistados referiu não conhecer esses direitos, e uma parcela menor ainda $(<6 \%)$ deles já havia recorrido à Justiça na busca de algum direito relativo à saúde. A baixa escolaridade contribui para esse desconhecimento. Também se pode apontar a circunstância de que um menor conhecimento sobre o direito à saúde afeta o grau de satisfação. Quanto maior o conhecimento, maior o nível de exigência e de identificação das falhas existentes nos serviços oferecidos, o que tende a levar a uma maior judicialização. Alguns estudos, porém, apontam para um aumento progressivo do conhecimento dos pacientes acerca de seus direitos, o que 
muitas vezes os leva à procura do Judiciário. Nos últimos anos, tem-se observado um aumento significativo de processos relacionados à demanda por medicações, incluindo aquelas que ainda não foram aprovadas pela Agência Nacional de Vigilância Sanitária (Anvisa) e de alto custo ${ }^{4}$. Em estudo recente realizado no Rio de Janeiro, entre julho de 2007 e junho de 2008, foram encontrados 281 processos relacionados a 144 medicamentos, sendo que a maioria deles $(66,6 \%)$ não estava incluída na lista de medicamentos da Anvisa ${ }^{5}$. Nesses casos, não se sabe se os pacientes haviam procurado o SUS em busca dos tratamentos já disponíveis na rede e se, em caso positivo, o que houve de errado.

Atualmente observa-se no Brasil um aumento no número de decisões judiciais obrigando o poder público a fornecer bens e serviços de saúde, fenômeno chamado de "judicialização da saúde"6. No Estado de São Paulo há uma taxa de sucesso do pleiteante contra o sistema público de saúde em torno de $85 \%$. Segundo Wang et al.,

O Judiciário brasileiro também tende a desconsiderar o impacto orçamentário de uma decisão judicial que obriga o sistema de saúde a fornecer um determinando tratamento. Para os juízes, em geral, questões relativas ao orçamento público, como a escassez de recursos e a não previsão de gasto, bem como o não pertencimento do medicamento pedido às listas de medicamentos do SUS, não são razões suficientes para se denegar o pedido de um tratamento médico, dado que este encontra respaldo no direito à saúde assegurado pela Constituição Federal.

Essa postura do Judiciário brasileiro permitiu - e até incentivou - o impressionante aumento no número de ações pleiteando tratamentos médicos com base no direito constitucional à saúde. O gasto do Ministério da Saúde com medicamentos cuja dispensação foi determinada por ordem judicial passou de R \$ 2,5 milhões em 2005 para aproximadamente R 266 milhões em 2011. No mesmo período, o número de medicamentos fornecidos judicialmente pela Secretaria de Saúde do Estado de São Paulo subiu de 799 para 14.563, um crescimento de $1.722,65 \%$ em cinco anos. Por outro lado, apesar da contribuição das decisões judiciais para a elevação no gasto com insumos de saúde para os

\footnotetext{
${ }^{4}$ FIGUEIREDO, Tatiana Aragão; OSÓRIO-DE-CASTRO, Claudia Garcia Serpa; PEPE, Vera Lúcia Edais. Evidencebased process for decision-making in the analysis of legal demands for medicines in Brazil. Cadernos de Saúde Pública, Rio de Janeiro, v. 29, supl. 1, p. S159-S166, 2013. Disponível em: <http://www.scielo.br/ pdf/csp/v29s1/a14.pdf>. http://dx.doi.org/10.1590/0102-311X00161812.

5ld. Ibid.

${ }^{6}$ WANG, Daniel Wei; VASCONCELOS, Natália Pires; OLIVEIRA, Vanessa Elias; TERRAZAS, Fernanda Vargas. Os impactos da judicialização da saúde no município de São Paulo: gasto público e organização federativa. Revista de Administração Pública, Rio de Janeiro, v. 48, n. 5, p. 1191-1206, 2014. Disponível em: <http:// www.scielo.br/pdf/rap/v48n5/06.pdf>. http://dx.doi.org/10.1590/0034-76121666.
} 
cidadãos brasileiros, observa-se um aumento expressivo nos últimos anos dos gastos pessoais com saúde. Boing et al., em estudo recente analisando dados da Pesquisa de Orçamentos Familiares de 2002-2003 (48.470 domicílios) e 2008-2009 (55.970 domicílios $)^{7}$, observaram aumento do chamado gasto catastrófico em saúde, definido como o dispêndio em saúde que excede um percentual pré-definido dos gastos totais ou da capacidade de pagamento do domicílio. Esses gastos variaram entre $0,7 \%$ e $21,0 \%$. As menores prevalências foram observadas em relação à capacidade de pagamento, enquanto as maiores, em relação ao total do consumo. Houve aumento de $25 \%$ na prevalência de gastos catastróficos em saúde de entre 2002-2003 e 2008-2009, quando utilizado o ponto de corte de 20\% em relação ao total de consumo; e de $100 \%$, quando aplicado o ponto de corte de $40 \%$ da capacidade de pagamento. Houve expressiva e crescente desigualdade socioeconômica na prevalência de gasto catastrófico em saúde no Brasil entre 2002-2003 e 2008-2009, chegando a ser 5,2 vezes maior o gasto catastrófico dos mais pobres e 4,2 vezes maior dos menos escolarizados.

Muito se debateu e se debate acerca da judicialização do acesso aos cuidados de saúde em nosso país. É reconhecido o elevado grau de interferência judicial na determinação do fornecimento de medicamentos e de outras prestações de saúde, afetando os orçamentos públicos e a equidade no atendimento de seus beneficiários, já que no Sistema Único de Saúde (SUS), que é público, recursos que se destinariam a áreas prioritárias e a pessoas mais carentes acabam sendo empregados para atender decisões que beneficiam indivíduos mais abonados e com maior acesso à informação ${ }^{8}$. A maioria dos entrevistados no presente estudo referiu já ter vivenciado pelo menos uma situação em que lhe fora prescrita medicação e a mesma não fora fornecida pelo SUS. A baixa escolaridade e o menor conhecimento acerca dos direitos relacionados à saúde parecem apontar, portanto, para uma menor procura do Judiciário e também para uma avaliação mais positiva acerca dos serviços prestados.

Os gastos públicos com a saúde no Brasil têm aumentado desde 2003, mas o ajuste pela inflação mostra uma redução líquida. Em 2007, apenas 8,4\% do Produto Interno Bruto (PIB) foi gasto com a saúde. Naquele ano, a proporção pública da despesa com saúde foi de $41 \%$, um valor baixo quando comparado a países como Reino Unido (82\%), Itália (77,2\%), Espanha (71,8\%), Estados Unidos

${ }^{7}$ BOING, Alexandra Crispim; BERTOLDI, Andréa Dâmaso; BARROS, Aluísio Jardim Dornellas; POSENATO, Leila Garcia; PERES, Karen Glazer. Desigualdade socioeconômica nos gastos catastróficos em saúde no Brasil. Revista de Saúde Pública, São Paulo v. 48, n. 4, p. 632-641, 2014. Disponível em: <http://www.scielo.br/ pdf/rsp/v48n4/pt_0034-8910-rsp-48-4-0632.pdf>. 10.1590/S0034-8910.2014048005111.

8VICTORA, Cesar; BARRETO, Maurício; LEAL, Maria do Carmo; MONTEIRO, Carlos; SCHMIDT Maria Inês; PAIM, Jairnilson; BASTOS, Francisco; ALMEIDA, Célia; BAHIA, Lígia; TRAVASSOS, Claudia; REICHENHEIM, Michael; BARROS, Fernando. Health in Brazil 6 - Health conditions and health-policy innovations in Brazil: the way forward. The Lancet, v. 377, n. 9782, p. 2042-2053, Jun. 2011. Disponivel em: <http://dx.doi. org/10.1016/S0140-6736(11)60055-X>. http://dx.doi.org/10.1016/S0140-6736(11)60055-X. 
$(45,5 \%)$ e México $(46,9 \%)^{9}$. Comparando-se os gastos com a saúde dos últimos governos do Brasil, no período de 1995 a 2012, observa-se um alinhamento de política, sendo ainda insuficientes para as demandas do país. Observa-se ainda uma tendência de queda acentuada da participação proporcional do Governo Federal nos gastos com saúde ao longo de todos os períodos. O percentual do gasto público per capita, em relação ao total gasto, está situado num patamar que não condiz com um sistema universal de saúde, estando distante de sistemas semelhantes, como ocorre em alguns países da Europa, e próximo do sistema privado americano ${ }^{10}$.

De acordo com a Lei n. 8080/1990 ${ }^{11}$, que dispõe sobre as condições para a promoção, proteção e recuperação da saúde, bem como a organização e o funcionamento dos serviços correspondentes, a iniciativa privada poderá participar do SUS em caráter complementar. O sistema de saúde do Brasil possui três subsetores: setor público (SUS), no qual os serviços são financiados e providos pelo Estado nos níveis federal, estadual e municipal; setor privado, no qual os serviços são financiados de diferentes maneiras com fundos públicos ou privados; e setor de saúde suplementar, constituído de planos de saúde com variados benefícios, taxas e subsídios ${ }^{12}$. A participação complementar de instituições privadas no SUS, prevista no artigo 199, parágrafo $1^{\circ}$ da Constituição Federal de $1988(\mathrm{CF} / 88)^{13}$, ocorre mediante elaboração de contrato de direito público ou convênio, com preferência para as entidades filantrópicas e sem fins lucrativos ${ }^{14}$.

No presente estudo, apenas dois pacientes referiram ter plano de saúde privado. Há uma tendência de expansão da saúde suplementar no Brasil. De acordo com dados da Agência Nacional de Saúde Suplementar (ANS), houve um crescimento de $18 \%$ a $25 \%$ entre 2003 e 2011 na porcentagem de pessoas cobertas por planos e seguros privados de saúde, sendo mais de $70 \%$ na modalidade de planos

9PAIM, Jairnilson; TRAVASSOS, Claudia; ALMEIDA, Célia; BAHIA, Lígia; MACINKO, James. op. cit., p. $1778-1797$.

${ }^{10}$ SOARES, Adilson; SANTOS, Nelson Rodrigues. Financiamento do Sistema Único de Saúde nos governos FHC, Lula e Dilma. Saúde em Debate, Rio de Janeiro, v. 38, n. 100, p. 18-25, 2014. Disponivel em: <http://www.scielo.br/pdf/sdeb/v38n100/0103-1104-sdeb-38-100-0018.pdf>. 10.5935/0103-104.20140002.

${ }^{11}$ BRASIL. Lei n. 8.080, de 19 de setembro de 1990. Dispõe sobre as condições para a promoção, proteção e recuperação da saúde, a organização e o funcionamento dos serviços correspondentes e dá outras providências. Disponível em: <http://www.planalto.gov.br/ccivil_03/leis/L8080.htm>. Acesso em: 09 set. 2016.

${ }^{12}$ PAIM, Jairnilson; TRAVASSOS, Claudia; ALMEIDA, Célia; BAHIA, Lígia; MACINKO, James. op. cit., p. 1778-1797.

13“A Art. 199. A assistência à saúde é livre à iniciativa privada. $\S 1^{\circ}$ As instituições privadas poderão participar de forma complementar do sistema único de saúde, segundo diretrizes deste, mediante contrato de direito público ou convênio, tendo preferência as entidades filantrópicas e as sem fins lucrativos." BRASIL. Constituição da República Federativa do Brasil de 1988. Disponível em: <http://www.planalto.gov.br/ ccivil_03/constituicao/constituicaocompilado.htm>. Acesso em: 09 set. 2016.

${ }^{14}$ Luciana Biondo. Parcerias público-privadas para consolidação do SUS. In: PEREIRA, Maurício Fernandes; COSTA, Alexandre Marino; MORITZ, Gilberto de Oliveira; BUNN, Denise Aparecida (Orgs.). Contribuições para a Gestão do SUS. Florianópolis: Fundação Boiteux, 2013. p. 158-177. 
coletivos empresariais, ou seja, como benefícios trabalhistas ${ }^{15}$. No Estado de São Paulo, $41 \%$ da população era coberta por planos de saúde no ano de $2013^{16}$.

A saúde suplementar, sem dúvida, é importante para atender à demanda que o SUS não consegue atender. Entretanto, o setor privado também enfrenta alguns problemas. Estudo recente foi realizado em São Paulo, fruto de uma parceria entre a Associação Paulista de Medicina e o Instituto Datafolha, para avaliação da satisfação dos usuários de planos de saúde ${ }^{17}$. Foram entrevistados 861 pacientes maiores de 18 anos que haviam utilizado algum plano de saúde nos últimos 24 meses. O maior índice de problemas relatados foi em relação ao pronto-atendimento ( $80 \%$ dos usuários que necessitaram de pronto-atendimento relataram algum problema), seguido de problemas relacionados a consultas médicas (66\%), sendo relatada principalmente demora para marcação de consultas. Em relação à realização de exames diagnósticos, $47 \%$ tiveram algum tipo de problema, incluindo demora para marcação de exames ou procedimentos e poucas opções de laboratórios ou clínicas especializadas. Apenas 15\% dos entrevistados relataram já ter feito alguma reclamação contra os planos de saúde, sendo que o principal motivo foi a recusa de realização de cirurgia e apenas $2 \%$ desses usuários recorreram à Justiça. Existem normas e resoluções elaboradas para mediar os conflitos entre as operadoras de saúde suplementar no Brasil, sob a supervisão da ANS. A Resolução Normativa n. 226/2010, criou uma alternativa junto ao consumidor e às operadoras para a resolução de divergências. Com a criação da Notificação de Investigação Preliminar (NIP), possibilitou-se a resolução de problemas relacionados a coberturas de planos em tempo estimado de dez dias. Referida resolução foi substituída pela Resolução Normativa 388/2015 ${ }^{18}$, que trouxe alguns contornos especiais, como a ampliação das matérias que podem ser discutidas e a publicização de todos os atos via internet. Essa nova resolução, visa ao diálogo entre operadoras e clientes como fase auxiliar e pré-processual, diminuindo assim o confronto pela via judicial.

Não se pode deixar de referir a possibilidade de buscar evitar ou minimizar a judicialização no campo da saúde por meio do recurso à Justiça Restaurativa. Pensada originariamente como forma de desafogar o Poder Judiciário quanto ao julgamento de crimes de menor potencial ofensivo e atos infracionais

\footnotetext{
${ }^{15}$ CARVALHO, Regina; FORTES, Paulo; GARRAFA, Volnei. Reflexiones sobre la participación pública y privada en la asistencia a la salud. Salud Pública de México, Cuernavaca, v. 56, n. 2, p. 221-225, mar./abr. 2014. Disponível em: <http://www.scielo.org.mx/pdf/spm/v56n2/v56n2a10.pdf>. 10.1590/S0034-8910.2014048005111.

${ }^{16}$ Associação Paulista de Medicina. Avaliação dos planos de saúde pelos usuários. São Paulo, ano 2, 2013. Disponível em: <http://www.apm.org.br/imagens/Pdfs/pesquisadatafolha.pdf>. Acesso em: 08 jan. 2015.

${ }^{17}$ Id. Ibid.

${ }^{18}$ AGÊNCIA NACIONAL DE SAÚDE SUPLEMENTAR. Resolução $n$. 388, de 25 de novembro de 2015. Dispõe sobre os procedimentos adotados pela Agência Nacional de Saúde Suplementar - ANS para a estruturação e realização de suas ações fiscalizatórias. Disponível em: <http://www.ans.gov.br/component/legislacao/ ?view=legislacao\&task=TextoLei\&format=raw\&id=MzEzNg==>. Acesso em: 11 set. 2016.
} 
praticados por crianças e adolescentes ${ }^{19}$, a Justiça Restaurativa caracteriza-se pela busca de um diálogo entre as partes envolvidas (vítima e autores de atos de violência). Busca-se uma mediação entre infrator e vítima, pelo diálogo, pelo qual afloram todas as dimensões do problema, inclusive aquelas não previstas pela norma jurídica. Mais do que aplicar uma sanção penal ou uma medida socioeducativa, busca-se resolver o conflito de maneira menos formal e mais efetiva. Podem-se perfeitamente imaginar cenários em que planos e seguros de saúde e seus usuários, no tocante à saúde suplementar, e mesmo instâncias do SUS e cidadãos interessados, no campo da saúde pública, possam, previamente ao ajuizamento de uma ação judicial, tentar pela via da mediação uma solução aceitável para o conflito.

Iniciativa interessante, nesse sentido, foi a criação, pelo Tribunal de Justiça do Estado de São Paulo, de um Núcleo de Apoio Técnico e de Mediação ${ }^{20}$, que atua previamente à apreciação de liminares e tutelas de urgência em ações movidas contra planos e seguros de saúde, visando inicialmente a obter uma composição amigável - e, não sendo possível, subsidiar os magistrados com informações técnicas necessárias ao melhor julgamento do processo. Instalado com a participação do mencionado tribunal, da ANS, da Associação Brasileira de Medicina de Grupo (Abramge) e da Federação Nacional de Saúde Suplementar (Fenasaúde), o Núcleo atuará inicialmente por meio da oferta, em até 24 horas, de uma proposta de composição amigável para o processo. Não se alcançando tal composição, o Núcleo encaminhará informações técnicas para os juízes. Assim, além da possibilidade de resolução de conflitos de forma célere e fora do Judiciário, possibilita, na eventualidade de não se chegar a um acordo, a oferta de elementos técnicos que permitirão decisões mais bem fundamentadas.

Em pesquisa realizada em cinco municípios do Estado de São Paulo foram entrevistados 471 usuários dos serviços de saúde públicos (SUS). Para $72 \%$ desses entrevistados, os serviços de saúde prestados estão resolvendo os problemas e as necessidades da população. Com base no acesso ao atendimento, 57,6\% da população queixou-se da presença de filas para o atendimento e 54,5\% afirmaram que existe uma demora excessiva entre o dia de agendamento da consulta e/ou exame até o dia do atendimento. Mais da metade $(69,5 \%)$ afirmou ter confiança na equipe de saúde; $92 \%$ já haviam recebido prescrição de medicamentos no SUS e, para $83,1 \%$ destes, os profissionais explicaram a administração do remédio; $73 \%$ relataram ter recebido atenção adequada a suas queixas por parte dos profissionais. Em relação

\footnotetext{
${ }^{19}$ CONSELHO NACIONAL DE JUSTIÇA. Justiça Restaurativa: o que é e como funciona. 24 nov. 2014. Disponível em: <http://www.cnj.jus.br/noticias/cnj/62272-justica-restaurativa-o-que-e-e-como-funciona>. Acesso em: 13 ago. 2015.

${ }^{20}$ TJSP, ANS, Abramge e FenaSaúde unidos para resolver, em 24 horas, questões que envolvem a assistência à saúde suplementar. Disponível em: <http://www.tjsp.jus.br/institucional/canaiscomunicacao/noticias/ Noticia.aspx?ld=26272>. Acesso em: 12 jun. 2015.
} 
aos serviços de saúde, $61,7 \%$ classificaram-nos como ótimos ou bons ${ }^{21}$. Em outro estudo, realizado em Foz do Iguaçu, no Paraná, foram entrevistados 99 usuários do serviço público de fisioterapia, que consideraram os serviços oferecidos como ótimos (todos os entrevistados). Observou-se, ainda, analogia significativa entre as dimensões relação terapeuta/paciente e marcação de consulta $(\mathrm{p}<0,05)$ e entre a marcação de consulta e o retorno/recomendação da clínica $(\mathrm{p}<0,05)$; e uma relação extremamente significante entre a marcação de consulta e o acesso ${ }^{22}$.

No Nordeste do Brasil ainda existem poucos estudos sobre a satisfação dos usuários dos serviços de saúde, tanto públicos quanto privados. Veras, em estudo transversal realizado na cidade de Fortaleza ${ }^{23}$ (Ceará), entrevistou 292 usuários do sistema de Ouvidoria em Saúde da Secretaria de Saúde do Estado do Ceará. O principal motivo relatado para a procura do serviço foi reclamar da qualidade do atendimento $(64,7 \%)$, seguido de orientação $(12,3 \%)$ e elogio $(11,5 \%)$. Os principais motivos de reclamação foram: falta de atendimento no serviço de saúde $(57,8 \%)$, mau atendimento no serviço de saúde (52\%) e falta de medicamento nas farmácias dos serviços de saúde $(8,6 \%)$. Problemas de falta de higiene nos serviços de saúde $(9,2 \%)$ e de limpeza nas ruas $(16,3 \%)$ também foram relatados. Com relação à própria Ouvidoria em Saúde, embora 48,7\% dos usuários estivessem satisfeitos com o serviço, as seguintes falhas foram citadas: falta de autonomia (14,7\%), demora na solução dos problemas (8\%) e poucos ouvidores (6,6\%). O difícil acesso ao serviço, a falta de interesse e o descaso com o problema dos usuários também foram citados.

No presente estudo, todos os pacientes entrevistados disseram estar satisfeitos com o serviço avaliado e a maioria qualificou o serviço como excelente (66\%). O serviço avaliado é de iniciativa privada e tem convênio com o SUS. Mesmo considerando que a baixa escolaridade e o reduzido grau de conhecimento de direitos revelado pelos entrevistados possam ter contribuído para uma avaliação mais favorável, tem-se que os serviços de saúde da iniciativa privada no Brasil, em geral, apresentam uma boa qualidade - muitos deles buscam a excelência no atendimento, inclusive com certificações de qualidade, como a ISO (International Organization for Standardization). A parceria entre o público e o privado pode ser benéfica no sentido de atender a uma

\footnotetext{
${ }^{21}$ MOIMAZ, Suzely Adas Saliba; MARQUES, Jeidson Anônio Morais; SALIBA, Orlando; GARBIN, Cléa Adas Saliba; ZINA, Lívia Guimarães; SALIBA, Nemre Adas. Satisfação e percepção do usuário do SUS sobre o serviço público de saúde. Physis: Revista de Saúde Coletiva, Rio de Janeiro, v. 20, n. 4, p. 1419-1440, 2010. Disponível em: <http://www.scielo.br/pdf/physis/v20n4/a19v20n4.pdf>. http://dx.doi.org/10.1590/S0103-73312010000400019.

${ }^{22}$ FRÉZ, Andersom Ricarco; NOBRE, Maria Inês Rubo de Souza. Satisfação dos usuários dos serviços ambulatoriais de fisioterapia da rede pública. Fisioterapia em Movimento, Curitiba, v. 24, n. 3, p. 419-428, jul./set. 2011. Disponível em: <http://www.scielo.br/pdf/fm/v24n3/06.pdf>. http://dx.doi.org/10.1590/S0103-51502011000300006.

${ }^{23}$ VERAS, Mirella Maria Soares. A satisfação dos usuários do SUS com o serviço de ouvidoria em saúde de Fortaleza. 158p. Dissertação (Mestrado em Saúde Pública) - Universidade Federal do Ceará. Fortaleza, 2005. Disponivel em: <http://www.repositorio.ufc.br/bitstream/riufc/1339/1/2005_dis_mmsveras.pdf>. Acesso em: 08 jan. 2015.
} 
demanda por serviços, sobretudo consultas médicas e realização de exames diagnósticos, que o setor público isoladamente não consegue suprir. É importante a discussão de como ampliar e melhorar as parcerias entre o SUS e os serviços privados de saúde, sobretudo em tempos de crise financeira, em que as contas públicas enfrentam grandes problemas. Um exemplo de bons resultados da parceria entre o setor público e o privado foi relatado recentemente pelo Hospital Israelita Albert Einstein (HIAE), em São Paulo. Foram analisados os resultados da parceria entre o referido hospital e o SUS em cirurgias vasculares realizadas entre abril de 2012 e julho de 2013. Todos os pacientes incluídos no estudo haviam sido submetidos à cirurgia e permanecido pelo menos um dia na unidade de terapia intensiva do HIAE. Um total de 62 procedimentos endovasculares foi realizado em 57 pacientes do SUS. Os custos hospitalares, incluindo as próteses utilizadas, foram significativamente maiores do que os valores pagos pelo SUS. Todos os procedimentos realizados obtiveram sucesso, e o tempo de espera entre a indicação do procedimento e a realização da cirurgia foi significativamente reduzido. Os autores concluíram que as parcerias público-privadas representam uma alternativa eficaz para acelerar a assistência e para melhorar o prognóstico de pacientes com doenças vasculares, o que minimiza o déficit estrutural do SUS. Os hospitais privados, que em geral encontram-se bem equipados, podem absorver parte da demanda pública por procedimentos cirúrgicos ${ }^{24}$. Outra parceria de sucesso foi reportada por Barbosa et al., mostrando o exemplo da cidade de Florianópolis, em Santa Catarina: entre os anos de 2008 e 2011, vários editais para a contratação de serviços de saúde privados aumentaram a oferta de serviços para a população. Em 2010 foi lançada a campanha "fila zero", tendo sido firmados 12 novos contratos, ampliando especificamente os serviços de patologia clínica, diagnóstico por anatomia patológica, densitometria óssea, diagnóstico em medicina nuclear e litotripsia extracorpórea ${ }^{25}$. A parceria público-privada ainda é pouco utilizada, como mostram Romano e Scatena na experiência da região de Cuiabá, no Mato Grosso ${ }^{26}$. No ano de 2011, a maioria dos estabelecimentos prestadores de assistência ambulatorial na baixada cuiabana (61,3\%) pertencia ao setor público, enquanto os prestadores privados/filantrópicos representavam 38,7\%. Na análise dos estabelecimentos que prestavam serviços auxiliares de diagnóstico e terapia (SADT), os prestadores privados/filantrópicos associados ao SUS detinham $65,5 \%$ desses estabelecimentos. Os autores pontuam, ainda, que o

\footnotetext{
${ }^{24}$ MENDES, Cynthia de Almeida; MARTINS, Alexandre de Arruda; TEIVELIS, Marcelo Passos; KUZNIEC, Sérgio; WOLOSKER, Nelson. Public private partnership in vascular surgery. Einstein, São Paulo, v. 12, n. 3, p. 342-346, 2014. Disponível em: <http://www.scielo.br/pdf/eins/v12n3/1679-4508-eins-12-3-0342.pdf>. http://dx.doi.org/10.1590/S1679-45082014GS3029.

${ }^{25}$ BARBOSA, Waldileuza Ferreira Rodrigues; OLIVO, Luis Carlos Cancellier; SILVA, Maria Luciana Biondo. Parcerias publico-privadas para consolidação do SUS. In: PEREIRA, Mauricio Fernandes; COSTA, Alexandre Marino; MORITZ, Gilberto de Oliveira; BUNN, Denise Aparecida (Orgs.). Contribuições para a gestão do SUS. Florianopolis: Fundação Boiteux, 2013. p. 158-177.

${ }^{26}$ ROMANO, Cátia Maria Costa; SCATENA, João Henrique Gurtler. A relação público-privada no SUS: um olhar sobre a assistência ambulatorial de média e alta complexidade na região de saúde da Baixada Cuiabana em Mato Grosso. Revista de Administração Pública, Rio de Janeiro, v. 48, n., p. 439-458, 2014. Disponível em: <http://www.scielo.br/pdf/rap/v48n2/a08v48n2.pdf>. http://dx.doi.org/10.1590/0034-76121618.
} 
número de estabelecimentos privados e filantrópicos existentes na região é grande e apenas uma pequena parcela deles presta serviços ao SUS. Não estavam vinculados ao SUS $88,3 \%$ dos estabelecimentos prestadores de assistência ambulatorial e 73,8\% dos prestadores de SADT.

\section{Conclusão}

Os usuários do Núcleo de Assistência Médica Integrada da Universidade de Fortaleza, um serviço da iniciativa privada em convênio com o SUS, se mostraram satisfeitos com os serviços oferecidos e consideraram o serviço como bom ou excelente. Provavelmente devido à baixa renda da maioria dos entrevistados, apenas uma minoria possuía plano de saúde. Os principais problemas evidenciados foram a dificuldade de acesso à medicação prescrita pelo SUS e a falta de conhecimento dos direitos relacionados à saúde. Foi observada uma baixa procura dos usuários entrevistados pelo Judiciário para questões ligadas à saúde, o que pode decorrer da baixa escolaridade e do reduzido grau de conhecimento de seus direitos demonstrados pela maior parte deles. Pode-se afirmar que, quanto maior esse grau de conhecimento, maior tende a ser a procura de meios judiciais, principalmente considerando a possibilidade de recorrer à Defensoria Pública, sem dispêndio com advogados particulares. Tal reduzido grau de conhecimento pode também explicar o elevado nível de satisfação demonstrado, já que o nível de exigência tende a aumentar com o maior acesso a informações, muito embora os problemas relatados com relação ao acesso a medicamentos não tenham ocorrido no âmbito do NAMI. Como o aumento do conhecimento quanto aos direitos pode levar a um crescimento da judicialização no tocante à saúde, mostra-se importante buscar meios alternativos de resolução de conflitos nesse campo. A mediação empreendida pela ANS, na forma da Resolução Normativa 388/2015, e a criação pelo Tribunal de Justiça do Estado de São Paulo do Núcleo de Apoio Técnico e de Mediação são experiências que merecem ser acompanhadas, avaliadas e eventualmente reproduzidas, com as devidas adaptações, inclusive no campo da saúde pública.

\section{Referências}

ASSOCIAÇÃO PAULISTA DE MEDICINA. Avaliação dos planos de saúde pelos usuários. São Paulo, ano 2, 2013. Disponível em: <http://www.apm.org.br/imagens/Pdfs/pesquisadatafolha.pdf $>$. Acesso em: 08 jan. 2015.

BARBOSA, Waldileuza Ferreira Rodrigues; OLIVO, Luís Carlos Cancellier; SILVA, Maria Luciana Biondo. Parcerias público-privadas para consolidação do SUS. In: PEREIRA, Maurício Fernandes; COSTA, Alexandre Marino; MORITZ, Gilberto de Oliveira; BUNN, Denise Aparecida (Orgs.). Contribuições para a gestão do SUS. Florianópolis: Fundação Boiteux, 2013. p. 158-177. 
BOING, Alexandra Crispim; BERTOLDI, Andréa Dâmaso; BARROS, Aluísio Jardim Dornellas; POSENATO, Leila Garcia; PERES, Karen Glazer. Desigualdade socioeconômica nos gastos catastróficos em saúde no Brasil. Revista de Saúde Pública, São Paulo v. 48, n. 4, p. 632-641, 2014. Disponível em: <http://www.scielo.br/pdf/rsp/v48n4/pt_0034-8910rsp-48-4-0632.pdf>. 10.1590/S0034-8910.2014048005111.

CARVALHO, Regina; FORTES, Paulo; GARRAFA, Volnei. Reflexiones sobre la participación pública y privada en la asistencia a la salud. Salud Pública de México, Cuernavaca, v. 56, n. 2, p. 221-225, mar./abr. 2014. Disponível em: <http://www.scielo.org.mx/pdf/spm/v56n2/ v56n2a10.pdf>. 10.1590/S0034-8910.2014048005111.

CONSELHO NACIONAL DE JUSTIÇA. Justiça Restaurativa: o que é e como funciona. 24 nov. 2014. Disponível em: <http://www.cnj.jus.br/noticias/cnj/62272-justica-restaurativao-que-e-e-como-funciona $>$. Acesso em: 13 ago. 2015.

ERVIN, Naomi. Does patient satisfaction contribute to nursing care quality? Journal of Nursing Administration, Philadelphia, v. 36, n. 3, p. 126-130, 2006.

FIGUEIREDO, Leonardo Vizeu. Curso de direito de saúde suplementar: manual jurídico de planos e seguros de saúde. 2. ed. Rio de Janeiro: Gen/Forense, 2012.

FIGUEIREDO, Tatiana Aragão; OSÓRIO-DE-CASTRO, Claudia Garcia Serpa; PEPE, Vera Lúcia Edais. Evidence-based process for decision-making in the analysis of legal demands for medicines in Brazil. Cadernos de Saúde Pública, Rio de Janeiro, v. 29, supl. 1, p. S159-S166, 2013. Disponível em: <http://www.scielo.br/pdf/csp/v29s1/a14.pdf>. http:// dx.doi.org/10.1590/0102-311X00161812.

FRÉZ, Andersom Ricarco; NOBRE, Maria Inês Rubo de Souza. Satisfação dos usuários dos serviços ambulatoriais de fisioterapia da rede pública. Fisioterapia em Movimento, Curitiba, v. 24, n. 3, p. 419-428, jul./set. 2011. Disponível em: <http://www.scielo.br/pdf/fm/v24n3/06. pdf>. http://dx.doi.org/10.1590/S0103-51502011000300006.

HAWTHORNE, Graeme; SANSONI, Jan; HAYES, Laura; MAROSSZEKY, Nick; SANSONI, Emily. Measuring patient satisfaction with health care treatment using the Short Assessment of Patient Satisfaction measure delivered superior and robust satisfaction estimates. Journal of Clinical Epidemiology, New York, v. 67, n. 5, p. 527-537, 2014. doi: 10.1016/j.jclinepi.2013.12.010.

INSTITUTO ECONÔMICO DE PESQUISA APLICADA. Sistema de Indicadores de Percepção Social-SIPS. 2. ed. (2011/2012). Assistência Social. Percepção sobre pobreza: causas e soluções. Brasília, DF; 2012. Disponível em: <http://www.ipea.gov.br/portal/images/stories/PDFs/ SIPS/111221_sips_assistenciasocial.pdf $>$. Acesso em: 07 jan. 2015.

KRAVITZ, Richard. Patient satisfaction with health care: critical outcome or trivial pursuit? Journal of General Internal Medicine, Philadelphia, v. 13, n. 4, p. 280-282, Apr. 1998. 
MENDES, Cynthia de Almeida; MARTINS, Alexandre de Arruda; TEIVELIS, Marcelo Passos; KUZNIEC, Sérgio; WOLOSKER, Nelson. Public private partnership in vascular surgery. Einstein, São Paulo, v. 12, n. 3, p. 342-346, 2014. Disponível em: <http://www.scielo.br/pdf/eins/ v12n3/1679-4508-eins-12-3-0342.pdf>. http://dx.doi.org/10.1590/S1679-45082014GS3029.

MERCER, Mary; HERNANDEZ-BOUSSARD, Tina; MAHADEVAN, Swaminatha; STREHLOW, Matthew. Physician identification and patient satisfaction in the emergency deparment: are they related? Journal of Emergency Medicine, New York, v. 46, n. 5, p. 711-718, May 2014. doi: 10.1016/j.jemermed.2013.08.036.

MOIMAZ, Suzely Adas Saliba; MARQUES, Jeidson Anônio Morais; SALIBA, Orlando; GARBIN, Cléa Adas Saliba; ZINA, Lívia Guimarães; SALIBA, Nemre Adas. Satisfação e percepção do usuário do SUS sobre o serviço público de saúde. Physis: Revista de Saúde Coletiva, Rio de Janeiro, v. 20, n. 4, p. 1419-1440, 2010. Disponível em: <http://www.scielo. br/pdf/physis/v20n4/a19v20n4.pdf>. http://dx.doi.org/10.1590/S0103-73312010000400019.

PAIM, Jairnilson; TRAVASSOS, Claudia; ALMEIDA, Célia; BAHIA, Lígia; MACINKO, James. The Brazilian health system: history, advances, and challenges. The Lancet, London, v. 377, n. 9779, p. 1778-1797, 2011. Disponível em: <http://www.thelancet.com/pdfs/journals/ lancet/PIIS0140-6736(11)60054-8.pdf>. http://dx.doi.org/10.1016/S0140-6736(11)60054-8.

ROMANO, Cátia Maria Costa; SCATENA, João Henrique Gurtler. A relação público-privada no SUS: um olhar sobre a assistência ambulatorial de média e alta complexidade na região de saúde da Baixada Cuiabana em Mato Grosso. Revista de Administração Pública, Rio de Janeiro, v. 48, n., p. 439-458, 2014. Disponível em: <http://www.scielo.br/pdf/rap/v48n2/ a08v48n2.pdf>. http://dx.doi.org/10.1590/0034-76121618.

SAVASSI, Leonardo Cançado Monteiro. A satisfação do usuário e a autopercepção da saúde em atenção primária. Revista Brasileira de Medicina de Família e Comunidade, Rio de Janeiro, v. 5, n. 17, p. 3-5, 2010. Disponível em: <https://www.rbmfc.org.br/rbmfc/article/ view/135/127>. http://dx.doi.org/10.5712/rbmfc5(17)135.

SOARES, Adilson; SANTOS, Nelson Rodrigues. Financiamento do Sistema Único de Saúde nos governos FHC, Lula e Dilma. Saúde em Debate, Rio de Janeiro, v. 38, n. 100, p. 18-25, 2014. Disponível em: <http://www.scielo.br/pdf/sdeb/v38n100/0103-1104-sdeb-38-100-0018. pdf>. doi: 10.5935/0103-104.20140002.

SOUZA, Renilson Rehem. O sistema público de saúde brasileiro. In: SEMINÁRIO INTERNACIONAL TENDENNCIAS E DESAFIOS DOS SISTEMAS DE SAÚDE NAS AMÉRICAS, (11-14 ago. 2002). Disponível em: <http://www.saude.sc.gov.br/ saudetrabalhador/conferencia_estadual/textos_apoio/O\%20Sistema\%20P\%FAblico $\% 20$ de\%20Sa\%FAde\%20Brasileiro\%28CEST\%29.pdf>. Acesso em: 07 jan. 2015.

TJSP, ANS, Abramge e FenaSaúde unidos para resolver, em 24 horas, questões que envolvem a assistência à saúde suplementar. Disponível em: <http://www.tjsp.jus.br/institucional/ canaiscomunicacao/noticias/Noticia.aspx?Id=26272>. Acesso em: 12 jun. 2015. 
VERAS, Mirella Maria Soares. A satisfação dos usuários do SUS com o serviço de ouvidoria em saúde de Fortaleza. 158p. Dissertação (Mestrado em Saúde Pública) - Universidade Federal do Ceará. Fortaleza, 2005. Disponível em: <http://www.repositorio.ufc.br/bitstream/ riufc/1339/1/2005_dis_mmsveras.pdf >. Acesso em: 08 jan. 2015.

VICTORA, Cesar; BARRETO, Maurício; LEAL, Maria do Carmo; MONTEIRO, Carlos; SCHMIDT Maria Inês; PAIM, Jairnilson; BASTOS, Francisco; ALMEIDA, Célia; BAHIA, Lígia; TRAVASSOS, Claudia; REICHENHEIM, Michael; BARROS, Fernando. Health in Brazil 6 - Health conditions and health-policy innovations in Brazil: the way forward. The Lancet, v. 377, n. 9782, p. 2042-2053, Jun. 2011. Disponível em: <http://dx.doi.org/10.1016/ S0140-6736(11)60055-X>. http://dx.doi.org/10.1016/S0140-6736(11)60055-X.

WANG, Daniel Wei; VASCONCELOS, Natália Pires; OLIVEIRA, Vanessa Elias; TERRAZAS, Fernanda Vargas. Os impactos da judicialização da saúde no município de São Paulo: gasto público e organização federativa. Revista de Administração Pública, Rio de Janeiro, v. 48, n. 5, p. 1191-1206, 2014. Disponível em: <http://www.scielo.br/pdf/rap/v48n5/06.pdf>. http:// dx.doi.org/10.1590/0034-76121666.

\section{Agradecimentos}

Agradecemos aos acadêmicos do curso de Medicina da Universidade de Fortaleza (Unifor): Lívia Studart de Meneses, Mariana Calíope Gonçalves, João Honorato Gorgonha Ribeiro Nóbrega e Antônio Carlos Chaves Gomes Filho, pela realização de parte das entrevistas com os pacientes do Núcleo de Atenção Médica Integrada (NAMI); e ao diretor do NAMI-Unifor, Rodrigo Dornfeld Escalante, pelo apoio a nossa pesquisa.

Geraldo Bezerra da Silva Junior - Pós-Doutorando em Saúde Coletiva pela Universidade Federal da Bahia; doutor e mestre em Ciências Médicas pela Universidade Federal do Ceará; graduado em Medicina pela Universidade Federal do Ceará (2005). Professor adjunto do Curso de Medicina e do Programa de Pós-Graduação em Saúde Coletiva da Universidade de Fortaleza. Fortaleza/CE, Brasil.E-mail: geraldobezerrajr@yahoo.com.br.

Eduardo Rocha Dias - Doutor em Direito pela Universidade de Lisboa; mestre em Direito pela Universidade Federal do Ceará; graduado em Direito pela Universidade Federal do Ceará. Professor do Programa de Pós-Graduação em Direito da Universidade de Fortaleza. Procurador Federal da Advocacia-Geral da União. Fortaleza/CE, Brasil. E-mail: eduardordias@hotmail.com. 